The Egyptian Journal of Hospital Medicine (April 2019) Vol. 75 (5), Page 2934-2941

\title{
Effect of Sildenafil Citrate When Added to Low Molecular Weight Heparin and Small Dose Aspirin on Uteroplacental Perfusion in Cases of High-Risk Pregnancy Asem A. Abdo Mousa ${ }^{(1)}$, Mohamed A. Mohamed ${ }^{(1)}$, Mohamed S. Radwan ${ }^{(2)}$, Amr M. Sholkamy \\ (1) Department of Obstetrics and Gynecology, (2) Department of Clinical Pathology, Faculty of Medicine, Al-Azhar UniversityCorrespondence to: Amr M Sholkamy, Mobile: (+20)01012347145; E-Mail: amr_sholkamy@yahoo.com
}

\begin{abstract}
Background: incubation with sildenafil citrate limits the effects of vasoconstrictors on myometrial small arteries of normal pregnancy women and pregnant women with fetal growth restriction. So that phosphodiesterase inhibitors seem to improve uterine perfusion safely in pregnancies with fetal growth restriction.

Objective: the main aim of this study was to demonstrate the effect of sildenafil citrate when added to low dose aspirin and low molecular weight heparin on the uterine and umbilical artery Doppler to improve neonatal outcome in patients with high risk pregnancy.

Subjects and Methods: the study was conducted at Al-Azhar University Hospitals, Cairo, Egypt. From March 2017 to January 2019 . One hundred patients (50 in each arm of the study), who had or have a high-risk pregnancy, were selected and enrolled into a randomized clinical trial.

Results: there was significant increase in amniotic fluid index (AFI) and umbilical artery Doppler parameters. Group B (heparin/aspirin) also showed significant improvement in fetal biometry and Doppler parameters but when comparing the differences between the two groups, none of these changes were statistically significant except for the femur length measurement and the uterine artery resistance index.

Conclusion: from the results of our study we concluded that heparin and aspirin combination or when sildenafil is also added have beneficial effects in the treatment of pregnant women whom vascular impairment is expected showing improvement in the fetal growth parameters and reduction of admission.
\end{abstract}

Keywords: sildenafil -heparin - aspirin - Outcome.

\section{INTRODUCTION}

Pregnancy is considered an acquired hypercoagulable state due to increased concentration of coagulation factors, decreased levels of anticoagulants and decreased fibrinolytic capacity ${ }^{(1)}$. The gradual increase in hypercoagulability during normal pregnancy predisposes to venous thromboembolism such as deep vein thrombosis and pulmonary embolism and to gestational vascular complications, including recurrent pregnancy loss, intrauterine growth restriction (IUGR), preeclampsia and placental abruption. These adverse pregnancy outcomes affect up to $15 \%$ of gestations and are the major cause of maternal and fetal morbidity and mortality ${ }^{(2)}$. Anticoagulant therapy is indicated during pregnancy for the prevention and treatment of venous thromboembolism, systemic embolism in patients with mechanical heart valves and, in combination with aspirin, for prevention of recurrent pregnancy loss in women with antiphospholipid antibodies ${ }^{(3)}$.

It has been long recognized that in preeclampsia, a severe maternal endothelial dysfunction must be identical to placental pathology to that underlying fetal growth restriction (4). Vascular endothelial activation is also present in pregnancies with fetal growth restriction without preeclampsia ${ }^{(5)}$.
Pregnancies with fetal growth restriction are expected in elevated peripheral resistance in the maternal arterial system as seen in pregnancies with preeclampsia (6). A poor perinatal outcome is expected in pregnancies with high vascular resistance in uterine circulation, but the pregnancies in which the resistance values are normalized in the later trimesters have a significantly better outcome ${ }^{(7)}$.

In a normal pregnancy, the trophoblast produces nitric oxide (NO) which plays an important role in vasodilatation in the fetoplacental circulation to improve oxygen and nutritional supply to the fetus ${ }^{(8,9)}$. Nitric oxide relaxes arterial and venous smooth muscle potently and might inhibit platelets aggregation and adhesion. Nitric oxide donors, as vasodilating agents, must be the possible therapeutic approach for embryo development and fetus growth. The umbilical vein endothelial cells in FGR do not respond to chronic hypoxia, which may lead to fetoplacental vasoconstriction ${ }^{(10)}$. As a locally potent vasodilator, nitric oxide helps regulate perfusion by counter balancing the effects of other vasoactive agents ${ }^{(11)}$.

Moreover, increased circulating phosphodiesterase (PDE) activity is suspected in women with preeclampsia (12). In pregnancies with fetal growth restriction and without preeclampsia, a reversible increased myometrial 
arterial tone by phosphodiesterase inhibition has been reported in vitro ${ }^{(\mathbf{1 3})}$.

Sildenafil citrate is a selective inhibitor of cyclic guanosine monophosphate (cGMP)-specific phosphodiesterase (PDE)-5 leading to cyclic guanosine monophosphate (cGMP) accumulation and enhances the relaxation elicited by exogenous and neural-released nitric oxide in corpus cavernous ${ }^{(14)}$. Sildenafil citrate increases uterine blood flow and potentiates estrogen induced vasodilatation ${ }^{\left({ }^{15}\right)}$. Intravaginal administration of sildenafil in the success of in vitro fertilization describes no deleterious effects on mother and fetus. The natural killer cells activity and endometrial thickness were significantly changed after vaginal sildenafil therapy so it might be an interesting therapeutic option before conception in women with recurrent reproductive failure (16).

In an animal study on guinea pigs, low doses of sildenafil favored fetal tolerability to induced intrapartum asphyxia and high doses of sildenafil increased the fetal weight by 1.5 times ${ }^{(17)}$.

Improved growth of a fetus with growth restriction whose mother is treated with sildenafil for pulmonary hypertension is noted ${ }^{(18)}$. Incubation with sildenafil citrate limits the effects of vasoconstrictors on myometrial small arteries of normal pregnancy women and pregnant women with fetal growth restriction ${ }^{\left({ }^{(19)}\right.}$. So that phosphodiesterase inhibitors seem to improve uterine perfusion safely in pregnancies with fetal growth restriction.

Reduced flow / increased resistance in uterine and umbilical arteries, indicative of reduced uteroplacental flow in pregnancies with fetal growth restriction, was documented by non-invasive Doppler ultrasound velocimetry ${ }^{(20)}$.

To the best of our knowledge no study compared the effect of sildenafil citrate when added to heparin/aspirin combination in the management of high risk pregnancy and their effect on Doppler studies, hence the aim to conduct our study.

\section{MATERIAL AND METHODS}

This prospective randomized cohort clinical trial was conducted at the Department of Obstetrics and Gynecology, Al-Azhar University Hospitals, Cairo, Egypt from March 2017 to January 2019. The study was approved by the Ethics and Scientific Committees of Al-Azhar University. Patients were first verbally advocated and consented about the study and then agreed participants were given a written consent before to be included. Moreover; everyone had the right to be withdrawn from the study at any time according her own will.
A total sample size of 90 patients (45 per group) was calculated by using a formula for the difference in the mean of the proposed variables that would provide $80 \%$ power to detect a $5 \%$ difference at $\mathrm{a}=0.05$ and which assumed that $10 \%$ of women would not respond ${ }^{(21)}$. By this; we selected 100 pregnant patients having a high risk pregnancy. They were allocated and divided randomly via computer-generated random numeric tables prepared by a statistician with concealment of method option by the use of sealed opaque envelopes that were given to a third party (nurse) who assigned patients into 2 equal study arms. Group A n=40: (Sildenafil group): Each case received sildenafil citrate orally $20 \mathrm{mg}$ every 8 hours (respatio tablet, Pharma Egypt Group) started and continued till delivery and the dose was adjusted according to maternal perception of side effects; in addition to low molecular weight heparin daily according to the body weight at the booking visit. The dose was adjusted and small dose of aspirin $75 \mathrm{mg}$ once daily was given. Group B n=43: (Heparin/Aspirin group) where each case received low molecular weight heparin (clexane 20, 40, $60 \mathrm{mg}$, Sanofi Aventis Company) as subcutaneous injection once daily plus low dose aspirin $75 \mathrm{mg}$ (aspocid $75 \mathrm{mg}$ tablet, CID Pharmaceutical) orally once daily from the time of inclusion till delivery in addition to placebo three times daily. After recruitment; cases were subjected to fetal and maternal surveillance and the frequency was depending on the result of preliminary assessment.

Patients excluded from our study were those who had multiple pregnancies, any previous medication supposed to alter utero-placental blood flow, suspected chromosomal or fetal congenital anomalies documented by level II ultrasound examination, those who had maternal or fetal emergencies necessitating delivery at time of recruitment in the study, diastolic blood pressure more than $110 \mathrm{mmHg}$ and when the BMI was more than 34 that would halt full measurement of fetal biometrics and Doppler parameters.

Fetal surveillance on follow-up was depending on weekly fetal biometry via measuring abdominal circumference (AC), bi-parietal diameter (BPD), femur length (FL), estimated fetal weight (EFW), amniotic fluid index. Umbilical artery and middle cerebral artery Doppler indices were estimated at inclusion and then repeated twice weekly or more frequently if abnormal indices were encountered. Maternal surveillance was obtained by measuring blood pressure, urine test for proteinuria by dipsticks. Routine preoperative testing for the coagulation profile and the international normalized ratio were done

Appearance of drug side effects; flushing, light headache and orifices bleeding, were indications for immediate 
stoppage. Antenatal corticosteroid for lung maturation was administered routinely in patients who were after 24 weeks gestation to enhance lung maturity and in cases where sudden preterm labor would be decided. Data were collected for comparison between 2 groups and we used the data at inclusion in the study and the follow-up after 2 weeks of treatment. Cases who necessitated delivery before completing two weeks of treatment were excluded from the study. Primary outcome measures included increase in AC and EFW after 2 weeks of therapy, number of surviving neonates in each group and duration of stay in NICU.

\section{Statistical analysis}

Recorded data were analyzed using the statistical package for social sciences, version 20.0 (SPSS Inc., Chicago, Illinois, USA). Quantitative data were expressed as mean \pm standard deviation (SD). Qualitative data were expressed as frequency and percentage.

\section{The following tests were done:}

- Independent-samples t-test of significance was used when comparing between two means.

- Chi-square $\left(\mathrm{x}^{2}\right)$ test of significance was used in order to compare proportions between two qualitative parameters.
The confidence interval was set to $95 \%$ and the margin of error accepted was set to $5 \%$. The p-value was considered significant as the following:

- Probability (P-value)

- P-value <0.05 was considered significant.

- P-value $<0.001$ was considered as highly significant.

- P-value >0.05 was considered insignificant.

\section{RESULTS}

The study included 100 cases with high risk pregnancy, 50 cases in the sildenafil A group and 50 cases in the heparin/low dose aspirin B group. They met the inclusion criteria and received the planned treatment protocol. ten cases delivered before completing two weeks of treatment and follow-up and seven cases were withdrawn from the study for their own reason, so excluded from statistical analysis of data (10 cases in the A group and 7 cases in the B group). The two groups were comparable regarding maternal age, parity, presence of medical disease namely diabetes, systemic lupus erythematosus, hypertension, IUGR and preeclampsia ( $p$-value $>0.05)$, (Table 1$)$.

Concomitant medications taken and history of previous high risk pregnancy were taken also in considerations.

Table (1): Patients' characteristics of both studied groups.

\begin{tabular}{|l|l|l|l|}
\hline Variable & $\begin{array}{l}\text { Group A } \\
(\mathbf{n = 4 0})\end{array}$ & Group B (n=43) & p- value \\
\hline Maternal age (years + SD) & $27.6 \pm 5.1$ & $28.5 \pm 4$ & 0.37 \\
\hline Gravidity (median and range) & $4(1-6)$ & $3(1-8)$ & 0.8 \\
\hline Parity (median and range) & $1(0-3)$ & $2(0-5)$ & 0.06 \\
\hline MEDICAL RISK: & & \multirow{2}{*}{0.35} \\
With medical disease : & $22(55 \%)$ & $28(65.1 \%)$ & \\
DM & $7(17.5 \%)$ & $10(23.3 \%)$ & \\
HTN & $5(12.5 \%)$ & $6(14 \%)$ & \\
IUGR & $3(7.5 \%)$ & $5(11.6 \%)$ & \\
SLE & $2(5 \%)$ & $3(7 \%)$ & 0.8 \\
Pre -eclampsia & $5(12.5 \%)$ & $4(9.3 \%)$ & 0.9 \\
\hline Past History of high risk pregnancy as RPL & $18(45 \%)$ & $15(34.9 \%)$ & 0.12 \\
\hline Concomitant Medication taken & $18(45 \%)$ & $15(34.9 \%)$ & \\
\hline Gestational age (days since LMP and in weeks) & $212.1 \pm 19.1$ & $218 \pm 15.4$ & \\
& $(30.3 \pm 2.7)$ & $(31.1 \pm 2.1)$ & \\
\hline
\end{tabular}

Table (2) shows the difference in fetal biometry and in Doppler indices in sildenafil group A before and after treatment (Group A). There is significant increase in AC and EFW. Umbilical artery Doppler indices are improved and this appeared statistically significant despite worsening in 11 cases $(22 \%)$ that developed absent end diastolic flow. The changes in MCA Doppler indices were not significant $(p>0.05)$. There was a significant decrease in the uterine artery RI with absent notch. Amniotic fluid index significantly increased after treatment with sildenafil.

Table (2): Study variables in group A before and after treatment.

\begin{tabular}{|l|l|l|l|}
\hline Variable & Before treatment & 2 w after treatment & p-value \\
\hline
\end{tabular}


Effect of Sildenafil Citrate When Added to Low Molecular Weight Heparin...

\begin{tabular}{|l|l|l|l|}
\hline Fetal biometry: & & & \\
\hline BPD/mm & $64.1 \pm 6.2$ & $69.5 \pm 8.4$ & 0.001 \\
\hline FL/mm & $44.6 \pm 7.2$ & $49.4 \pm 7.6$ & 0.001 \\
\hline AC/mm & $188.7 \pm 40.5$ & $206.8 \pm 32.4$ & 0.002 \\
\hline EFW/gm & $926.8 \pm 453.2$ & $1105.3 \pm 429.3$ & 0.001 \\
\hline UA Doppler: & & & \\
S/D & $4.3(2.2-5.7)$ & $3.2(2.1-4.2)$ & 0.001 \\
PI & $1.6 \pm 0.6$ & $1.4 \pm 0.5$ & 0.001 \\
RI & $0.8 \pm 0.1$ & $0.7 \pm 0.1$ & 0.003 \\
AEDF & 0 & $11(27.5 \%)$ & 0.001 \\
\hline MCA Doppler: & & & \\
PI & $1.5 \pm 0.2$ & $1.3 \pm 0.3$ & 0.06 \\
RI & $0.6 \pm 0.3$ & $0.81 \pm 0.2$ & 0.055 \\
\hline Uterine artery & 0.67 & 0.51 & 0.005 \\
RI (cm/sec) & $(0.0-1.0)$ & $(0.00-0.83)$ & \\
\hline AFI cm & $4.3 \pm 1.1$ & $5.7 \pm 1.2$ & 0.001 \\
\hline
\end{tabular}

Group B showed a significant increase in fetal growth parameters after treatment as AC and EFW were $201 \mathrm{vs} 218$ (p $=0.03$ ) and $996 \mathrm{gm}$ vs 1187 ( $\mathrm{p=0.001)}$ respectively. Significant changes in umbilical artery S/D ratio PI but not RI as shown in (Table 4). Significant change in MCA PI was observed. Eight cases had absent EDF. There was a significant decrease in the uterine artery RI with absent notch. Also there was improvement in AFI ( $\mathrm{p}=0.001)$.

Table (3): Study variables in group B before and after treatment.

\begin{tabular}{|l|l|l|l|}
\hline Variable & Before treatment & 2w after treatment & $\mathrm{p}$-value \\
\hline Fetal biometry: & & & \\
BPD mm & & & \\
FL mm & $66.7 \pm 8.9$ & $70.5 \pm 8.8$ & 0.001 \\
AC mm & $48.4 \pm 9.3$ & $51.9 \pm 10.1$ & 0.001 \\
EFW gm & $201.3 \pm 43.7$ & $218.4 \pm 49.4$ & 0.03 \\
& $996.1 \pm 394$ & $1187.07 \pm 389.6$ & 0.001 \\
\hline UA Doppler: & & & \\
S/D & $3.9(2.4-5.5)$ & $3.4(2.5-5.3)$ & 0.001 \\
PI & $1.5 \pm 0.62$ & $1.3 \pm 0.54$ & 0.001 \\
RI & $0.7 \pm 0.13$ & $0.7 \pm 0.17$ & 0.025 \\
AEDF & 0 & $8(18.6 \%)$ & 0.002 \\
\hline MCA Doppler: & & & \\
PI & $1.3 \pm 0.5$ & $1.2 \pm 0.33$ & 0.02 \\
RI & $0.7 \pm 0.15$ & $0.72 \pm 0.2$ & 0.068 \\
\hline Uterine artery & 0.65 & 0.58 & \\
RI (cm/sec) & $(0.48-1.00)$ & $(0.37-0.78)$ & 0.001 \\
\hline AFI & $3.8 \pm 1.8$ & $5.6 \pm 0.69$ & 0.001 \\
\hline
\end{tabular}

There was no statistically significant difference between the two groups before starting treatment (Table 4) regarding fetal biometry (BPD, FL, AC and EFW) by ultrasound. Also Doppler indices were comparable; none of the two groups shows AEDF in umbilical artery.

The MCA Doppler was significantly different in the RI rather than the PI which showed insignificant difference ( $\mathrm{p}=0.04$ in $\mathrm{RI}$ and $\mathrm{p}=0.08$ in PI). There was insignificant difference between the 2 groups as regarding the RI of uterine artery with $\mathrm{p}=0.783$. Only AFI was significantly lower in the heparin treated group $(\mathrm{p}=0.04)$ and when comparing between the two groups after the treatment as regarding the fetal biometry found that there was no statistically significant difference except for the femur length measurement and the uterine artery resistance index (Table 5) . 
Asem Mousa et al.

Table (4): Comparison of study variables between the two groups before treatment

\begin{tabular}{|l|l|l|l|l|}
\hline & Variable & Group A & Group B & p -value \\
\hline Fetal biometry: & BPD/mm & $64.1 \pm 6.2$ & $66.7 \pm 8.9$ & 0.15 \\
& FL/mm & $44.6 \pm 7.2$ & $48.4 \pm 9.3$ & 0.08 \\
& AC/mm & $188.7 \pm 40.5$ & $201.3 \pm 43.7$ & 0.2 \\
& EFW'/gm & $926.8 \pm 453.2$ & $996.1 \pm 394$ & 0.45 \\
\hline UA Doppler: & S/D & $4.3(2.2-5.7)$ & $3.9(2.4-5.5)$ & 0.86 \\
& PI & $1.6 \pm 0.6$ & $1.5 \pm 0.62$ & 0.76 \\
& RI & $0.8 \pm 0.1$ & $0.7 \pm 0.13$ & 0.44 \\
& AEDF & 0 & 0 & \\
\hline MCA Doppler: & PI & $1.5 \pm 0.2$ & $1.3 \pm 0.5$ & 0.08 \\
& RI & $0.6 \pm 0.3$ & $0.7 \pm 0.15$ & 0.04 \\
\hline & Uterine artery & 0.67 & 0.65 & 0.783 \\
& RI (cm/sec) & $(0.0-1.0)$ & $(0.48-1.0)$ & \\
\cline { 2 - 5 } & AFI & $4.3 \pm 1.1$ & $3.8 \pm 1.8$ & 0.04 \\
\hline
\end{tabular}

Table (5): Comparison of study variables between the two groups after treatment.

\begin{tabular}{|l|l|l|l|}
\hline Variable & Group A & Group B & p -value \\
\hline Fetal biometry: & & & \\
BPD/mm & $69.5 \pm 8.4$ & $70.5 \pm 8.8$ & 0.09 \\
FL/mm & $49.4 \pm 7.6$ & $51.9 \pm 10.1$ & 0.04 \\
AC/mm & $206.8 \pm 32.4$ & $218.4 \pm 49.4$ & 0.06 \\
\hline EFW/gm & $1105.3 \pm 429.3$ & $1187.07 \pm 389.6$ & 0.3 \\
\hline UA Doppler: & & & \\
S/D & $3.2(2.1-4.2)$ & $3.4(2.5-5.3)$ & 0.5 \\
PI & $1.4 \pm 0.5$ & $1.3 \pm 0.54$ & 0.8 \\
RI & $0.7 \pm 0.1$ & $0.7 \pm 0.17$ & 0.6 \\
AEDF & $11(27.5 \%)$ & $8(18.6 \%)$ & 0.3 \\
\hline MCA Doppler: & & & \\
PI & $1.3 \pm 0.3$ & $1.2 \pm 0.33$ & 0.06 \\
RI & $0.81 \pm 0.2$ & $0.72 \pm 0.2$ & 0.19 \\
\hline Uterine artery & 0.51 & 0.58 & \\
RI(cm/sec) & $(0.00-0.83)$ & $(0.37-0.78)$ & 0.006 \\
\hline AFI & $5.7 \pm 1.2$ & $5.6 \pm 0.69$ & 0.4 \\
\hline
\end{tabular}

Table (6): Comparison of the outcome of the two groups.

\begin{tabular}{|l|l|l|l|}
\hline Variable & Group A n=(40) & Group B n= (43) & p - value \\
\hline Time to delivery (days) & $21.5 \pm 3.1$ & $25.6 \pm 2.8$ & 0.001 \\
\hline NICU admission (days) & $7(0-24)$ & $9(0-20)$ & 0.8 \\
\hline Survivals (alive) & $19(47.5 \%)$ & $26(60.5 \%)$ & 0.2 \\
\hline END & & & \\
\hline ND & $5(12.5 \%)$ & $9(20.9 \%)$ & 0.21 \\
\hline SB & $4(10 \%)$ & $3(7 \%)$ & 0.2 \\
\hline Side effects & $12(30 \%)$ & $5(11.6 \%)$ & 0.08 \\
\hline
\end{tabular}

The time between enrolment in the study and delivery was significantly longer in heparin group $(\mathrm{P}<0.001)$ and the sildenafil group experienced significant side effects (13 cases vs none). There were no differences in NICU admission, survivals, early neonatal death or neonatal deaths between the two groups.

\section{DISCUSSION}


Effect of Sildenafil Citrate When Added to Low Molecular Weight Heparin...

Our study was conducted to compare two modalities of therapies with different mechanisms directed to increase uteroplacental perfusion and thereafter improving perinatal outcome in patients whom vascular insufficiency is suspected, sildenafil citrate (a vasodilator drug) and the heparin/aspirin combination (antithrombotic drugs) Vs heparin/aspirin combination (antithrombotic drugs) alone .

The maternal characteristic of the studied groups were not significantly different specifically the maternal risk factors as all were expected to have vascular impairment.

Von Dadelszen et al. ${ }^{(22)}$ studied earlier gestational age $(22 \pm 4$ weeks) with high risk pregnancy specifically IUGR and evaluated the effect of sildenafil on severe early onset IUGR but our patients in both groups were at more than 30 weeks ( $30.3 \pm 2.7$ in Group A vs. $31.1 \pm 2.1$ in Group B). This may be explained by the fact that we selected elder gestational age due to poor neonatal outcome in our country with a similar earlier gestational age.

Our results also proved a significant increase in fetal growth measurements and estimated fetal weight after sildenafil citrate treatment and this comes in accordance with that conducted by Von Dadelszen $\boldsymbol{e t} \boldsymbol{a l}$. ${ }^{(22)}$ who documented an improving effect of sildenafil on fetal growth measurements mainly AC and EFW versus no treatment.

The dosage form, which was $25 \mathrm{mg}$ three times daily was equally effective as single dose of $50 \mathrm{mg}$ per day as randomized double blinded and placebo controlled trial by Dastjerdi et al. ${ }^{(23)}$, who examined 41 pregnant women with documented FGR at 24-37 weeks of gestation and evaluated them for the effect of a single dose of $50 \mathrm{mg}$ of SC on uteroplacental circulation by Doppler US of the UA and MCAs. They found that the sildenafil group fetuses demonstrated a significant decrease in S/D ratios [0.60 (SD: 0.40) (95\% CI: 0.37$0.84), P<0.001]$ and PI (0.12 (SD: 0.15$)$ (95\% CI: $0.02-$ $0.22), P=0.019]$ for the UA and a significant increase in MCA PI [0.51 (SD: 0.60) (95\% CI: 0.16-0.85), $P=$ 0.008]. They concluded that Doppler velocimetry index values reflect decreased placental bed vascular resistance after sildenafil administration.

Another case control study of pregnant female with FGR, sildenafil citrate $50 \mathrm{mg}$ tid was used. Doppler showed improved utero-placental blood flow with estimated fetal weight of around $800 \mathrm{gm}$ and hence pregnancy could be continued to another 3 weeks. A live male baby of 800 gram was delivered and shifted to Neonatal Intensive Care Unit (NICU) for further management. After 80 days of NICU care, the baby was finally discharged healthy with a weight of $2.3 \mathrm{~kg}$. After
1 month of discharge, mother and infant came for follow up and both were doing perfectly well. Also, the infant was checked by pediatrician and was found healthy ${ }^{(24)}$.

Another important finding in our results was the effect of sildenafil citrate on placental perfusion evaluated by changes in umbilical artery Doppler and middle cerebral artery Doppler where significant changes in Doppler indices occurred after treatment as shown in Table (3).

This latter finding comes in accordance with a study investigated also the effect of sildenafil citrate on utero-placental perfusion in cases of high risk pregnancies done by Marzieh et al. ${ }^{(25)}$ and Samangaya et al. ${ }^{(26)}$.

In contrary to our study, the maternal sildenafil for severe fetal growth restriction (STRIDER): a multicentre, randomized, placebo-controlled, double-blind trial Xiaoyuan et al. ${ }^{(27)}$ was done between Nov 21, 2014, and July 6, 2016, recruited 135 women and randomly assigned 70 women to sildenafil and 65 women to placebo. No difference was found in the median randomization to delivery interval between women assigned to sildenafil (17 days [IQR 7-24]) and women assigned to placebo (18 days [8-28]; $p=0.23$ ). Live births (relative risk [RR] 1.06, $95 \%$ CI 0.84 to $1.33 ; \mathrm{p}=0-62)$, fetal deaths $(0.89,0.54$ to $1.45 ; \mathrm{p}=0.64)$, neonatal deaths $(1.33,0.54$ to 3.28 ; $\mathrm{p}=0.53)$, and birth weight $(-14 \mathrm{~g},-100$ to $126 ; \mathrm{p}=0.81)$ did not differ between groups. No differences were found for any other secondary outcomes. Eight serious adverse events were reported during the course of the study (six in the placebo group and two in the sildenafil group).

Sildenafil did not prolong pregnancy or improve pregnancy outcomes in severe early onset fetal growth restriction and therefore it should not be prescribed for this indication outside of research studies with explicit participants consent. In this study fetal growth restriction defined as a combination of estimated fetal weight or abdominal circumference below tenth percentile and absent or reversed end-diastolic blood flow in the umbilical artery on Doppler velocimetry, which is exclusion criteria in our study ${ }^{(\mathbf{1 2}, \mathbf{2 8})}$.

These findings were consistent with Miller et al. (12) who found in an experimental animal study that sildenafil reduced uterine blood flow and this was associated with significant deterioration in fetal wellbeing. They explained their findings by the action of sildenafil on maternal systemic circulation, altering it and resulting in blood flow steal from the utero-placental circulation to the systemic vascular circulation that lowered its resistance due to widespread systemic vasodilatation. This may be explained as there is extensive cellular and tissue distribution of the PDE enzyme throughout the body and therefore lack of relative specificity within the uteroplacental circulation. These 
findings of Miller et al. ${ }^{(12)}$ in the animal study couldn't be confirmed in human.

Interim data from an independent clinical trial, the Dutch STRIDER (Sildenafil Therapy in Dismal prognosis Early-onset fetal growth Restriction) study, suggest an increased risk of persistent pulmonary hypertension of the newborn (PPHN) and neonatal mortality when sildenafil was used in pregnancy for intrauterine (fetal) growth restriction compared with placebo. The group assigned to sildenafil had an incidence of 17 cases of PPHN in 64 babies (27\%), including 11 deaths before discharge. In the placebo group, 3 of 58 babies (5\%) had PPHN, with no reported deaths before discharge. These findings occurred in the absence of any benefit shown on the primary endpoint of neonatal survival until term age.

The Dutch STRIDER study was one of 5 independent studies by an international collaboration investigating the use of sildenafil for this unauthorized use. The 5 trials in the STRIDER Consortium were undertaken in the UK, Ireland, The Netherlands, New Zealand/Australia, and Canada (28). This study was the main cause for dropping of most cases in the current study once the patients had known of such thing.

It is worth noting that sildenafil can be used in pregnant women with pulmonary hypertension showing significant clinical improvement in the cardiac symptoms, function and hemodynamic parameters. It also significantly improved pregnancy outcome through reducing prematurity, increasing the birth weight and lowering the cesarean section rate ${ }^{(23)}$. Another important consideration when administering drugs in pregnancy is the large increase in circulating plasma volume, which may alter the drug pharmacokinetics of sildenafil. As yet, there is no cases reported with neonatal pulmonary hypertension developed in the study. The use of low molecular weight heparin with or without small dose aspirin to improve maternal and perinatal outcomes in cases at risk for placental insufficiency has been extensively studied in the past years on the assumption that heparin prevents placental infarctions with subsequent increased placental perfusion ${ }^{(29,30)}$.

In the current study, the second group that received heparin/aspirin combination showed a significant increase in fetal AC, EFW as well as acceptable changes in Doppler indices ( $p$-values $<0.05$ ). On the other hand, some other studies found no beneficial effect from the addition of heparin to the standard obstetric care in the view of increasing fetal weight or placental perfusion ${ }^{\mathbf{3 1} \text {, }}$ 32). This is not coping with the result of the current study.

To the best of our knowledge there is no study comparing the effects of the use of the previous medications in IUGR at least in our locality. Although our results show a significant improvement in fetal growth parameters and Doppler indices in each group separately but these changes are not significantly different when comparing the two groups together. In addition, the duration of admission to NICU and neonatal survival were not significantly different between both groups. Heparin/Aspirin treatment group showed prolongation of pregnancy than the sildenafil treated group also with significant side effects observed in the sildenafil-treated group. These side effects of sildenafil were also observed in many other studies but did not require discontinuation of the treatment ${ }^{(33)}$.

Despite our results seem promising and convenient, but there are some limitations and drawbacks of this study. These mainly include relatively low number included, being 50 cases only in each group, and also being two centers rather than multicenter study that would be more informative when gathering data and comparing all results.

From the results of our study we concluded that sildenafil, heparin and aspirin combination has beneficial effects in the treatment strategy of growth restricted fetuses rather than heparin and aspirin alone with improvement of fetal growth parameters and reduction of NICU admissions. None of the treatment options is found superior to the other, however sildenafil carries more side effects despite being tolerated.

\section{REFERENCES}

1. Stirling Y, Woolf L, North WR, Seghatchian MJ, Meade TW (2002): Haemostasis in normal pregnancy. Women's issues in thrombosis and hemostasis, Martin Dunitz Ltd., London, pp. 151-165.

2. Kovac MK, Lalic-Cosic SZ, Dmitrovic JM, Djordjevic VJ, Radojkovic DP (2015):Thrombin generation, D dimer and protein $\mathrm{S}$ in uncomplicated pregnancy. Clin Chem Lab Med., 53(12):1975-9.

3. Bates SM, Greer IA, Pabinger I et al. (2008): Venous thromboembolism, thrombophilia, antithrombotic therapy, and pregnancy: American College of Chest Physicians evidence-based clinical practice guidelines (8th ed.). Chest, 133: 844-886.

4. Gilbert JS, Verzwyvelt J, Colson D, Arany M, Karumanchi SA, Granger JP (2010): Recombinant vascular endothelial growth factor 121 infusion lowers blood pressure and improves renal function in rats with placental ischemia-induced hypertension. Hypertension, 55:380-5.

5. Johnson MR, Anim-Nyame N, Johnson P, Sooranna SR, Steer PJ (2002): Does endothelial cell activation occur with intrauterine growth restriction? BJOG., 109:836-9.

6. Schiessl B, Kainer F, Oberhofer R, Jundt K, Friese K (2006): Doppler sonography of the uterine and the cubital arteries in normal pregnancies, preeclampsia and intrauterine growth restriction: evidence for a systemic vessel involvement. J Perinat Med., 34:139-44. 
7. Soregaroli M, Valcamonico A, Scalvi L, Danti L, Frusca T (2001): Late normalisation of uterine artery velocimetry in high risk pregnancy. Euro J Obstet Gynecol Reprod Biol., 95:42-5.

8. Rosselli M, Keller RJ, Dubey RK (1998): Role of nitric oxide in the biology, physiology and pathophysiology of reproduction. Hum Reprod Update, 4:3-24.

9. Ramsay B, Sooranna SR, Johnson MR (1996): Nitric oxide synthase activities in human myometrium and villous trophoblast throughout pregnancy. Obstet Gynecol., 87:249-53.

10. Bewley S, Chard T, Grudzinskas G, Campbell S (1993): The relationship of uterine and umbilical Doppler resistance to fetal and placental protein synthesis in the second trimester. Placenta, 14:663-70

11.Nanetti L, Giannubilo SR, Rafiaelli F, Curzi CM, Vignini A, Moroni C et al. (2008): Nitric oxide and peroxynitrite platelet levels in women with small-forgestational-age fetuses. BJOG., 115:14-21.

12. Miller SL, Loose JM, Jenkin G, Wallace EM (2009): The effects of sildenafil citrate (Viagra) on uterine blood flow and well being in the intrauterine growth-restricted fetus. Am J Obstet Gynecology, 200(102):e1-7.

13. Wareing M, Myers JE, O'Hara M, Kenny LC, Taggart MJ, Skillern L et al. (2006): Phosphodiesterase-5 inhibitors and placental small artery function in normal pregnancy and pre-eclampsia. Eur J Obstet Gynecology Reprod Biol., 127:41-9.

14. Ballard SA, Gingell CJ, Tang K, Turner LA, Price ME, Naylor AM (1998): Effects of sildenafil on the relaxation of human corpus cavernosum tissue in vitro and on the activities of cyclic nucleotide phosphodiesterase isozymes. J Urol., 159:2164-71.

15.Zoma WD, Baker RS, Friedman A, Clark KE (2004): Sildenafil citrate (Viagra) increases uterine blood flow and potentiates estrogen-induced vasodilation. Am J Obstet Gynecol., 190:1291-7.

16.Jerzak M, Kniotek M, Mrozek J, Górski A (2008): Sildenafll increases successful pregnancies after recurrent miscarriage. Fertil Steril., 90:1848-53.

17. Sánchez-Aparicio P, Mota-Rojas D, Nava-Ocampo AA, Trujillo-Ortega ME, Alfaro- Rodríguez A, Arch E (2008): Effects of sildenafil on the fetal growth of guinea pigs and their ability to survive induced intrapartum asphyxia. Am J Obstet Gynecol., 198(127):1-6.

18. Lacassie HJ, Germain AM, Valdés G, Fernández MS, Allamand F, López H (2004): Management of Eisenmenger syndrome in pregnancy with sildenafil and Larginine. Obstet Gynecol., 103:1118-20.

19. Wareing M, Myers JE, O'Hara M, Baker PN (2005): Sildenafil citrate (Viagra) enhances vasodilatation in fetal growth restriction. J Clin Endocrinol Metab., 90:2550-5.

20. Bower S, Kingdom J, Campbell S (1998): Objective and subjective assessment of abnormal uterine artery Doppler flow velocity waveforms. Ultrasound Obstet Gynecol., 12:260-4.

21.Dean AG, Sullivan KM and Soe MM (2005): Open Epi: Open Source Epidemiologic Statistics for Public Health. www.OpenEpi.com .

22. Von Dadelszen P, Dwinnell S, Magee LA, Carleton BC, Gruslin A, Lee B (2011): Sildenafil citrate therapy for severe early-onset intrauterine growth restriction. BJOG., 118:624-628.

23.Dastjerdi MV, Hosseini S, Bayani L (2012): Sildenafil citrate and uteroplacental perfusion in fetal growth restriction. J Res Med Sci., 17:632-636.

24.Panda S, Das A, Nowroz H et al. (1994): Sildenafil citrate in fetal growth Patrono C. Aspirin as an antiplatelet drug. N Engl J Med., 330:1287-94.

25. Marzieh VD, Sayedehafagh $H$ and Leila $B$ (2012): Sildenafil citrate and uteroplacental perfusion in fetal growth restriction. J Res Med Sci., 17 (7): 632-6.

26. Samangaya RA, Mires G, Shennan A, Skill-Ern L, Howe D, Mcleod A (2009): A randomised, double-blinded, placebo-controlled study of the phos-phodiesterase type 5 inhibitor sildenafil for the treatment of preeclampsia. Hypertens Pregnancy, 28: 369-82.

27. Xiaoyuan S, Kefang W, Wang W, Li B (2014): STRIDER: a multicentre, randomised, placebo-controlled, clinical efficiency of sildenafil citrate in the treatment of pregnancy complicated with pulmonary hypertension :Chinese Journal of Obstetrics and Gynaecology, 49(06):414-418.

28.Sharp A, Cornforth C, Jackson R et al. (2018): Maternal sildenafil for severe fetal growth restriction (STRIDER): a multicentre, randomised, placebo-controlled, double-blind trial. Lancet Child Adolesc Health, 2: 93-102.

29. Dodd JM, Mcleod A, Windrim RC, King-Dom J (2018): Antithrombotic therapy for improving maternal or infant health outcomes in women considered at risk of placental dysfunction. Cochrane Database Syst Rev., 6: CD006780, 2010.

30.Singh S, Sinha R and Kaushik M (2016): Prophylactic Low Molecular Weight Heparin Improving Perinatal Outcome in Non-thrombophilic Placental-Mediated Complications. J Obstet Gynaecol India, 66 (6): 436- 40.

31.Katie MG, Lesley MM, Laura KM, Arier CL, Joanne MS (2017): Enoxaparin for the prevention of preeclampsia and intra-uterine growth restriction in women with a history: A randomized trial. Am J Obstet Gynecol., 216(3):296-297.

32.Haddad B, Winer N, Chitrit Y, Houfflin-Debarge V, Chauleur C, Bages K, Tsatsaris V, Benachi A, Bretelle F, Gris JC, Bastu-JI-Garin S (2016): Enoxaparin and Aspirin Compared With Aspirin Alone to Prevent PlacentaMediated Pregnancy Complications: A Randomized Controlled Trial. Ob Stet Gynecol., 128 (5): 1053-63.

33. Boyce EG and Umland EM (2001): Sildenafil citrate: A therapeutic update. Clin Ther., 23: 2-23. 